\title{
Is Ultrasound a Viable Imaging Modality for the Diagnosis of Hepatocellular Carcinoma? A Cross-Sectional Study
}

\author{
Reeta Rani ${ }^{1}$, Uzma Mumtaz ${ }^{2}$, Amjad Shah ${ }^{3}$, Aamna Qazi ${ }^{4}$, Afshan Shaikh ${ }^{5}$, Syed Muhammad Kashif ${ }^{6}$
}

\author{
1 Assistant Professor, Department of Radiology, Shaheed Mohtarma Benazir Bhutto Medical College, Lyari Pakistan \\ Contribution in the study \\ 2 Instructor, Department of Radiology, Sindh Government Lyari General Hospital, Karachi Pakistan \\ 2 Contribution in the study \\ 3 Senior Registrar, Department of Radiology, Shaheed Mohtarma Benazir Bhutto Medical College, Lyari Pakistan \\ Contribution in the study \\ Resident Medical Officer, Medical Unit III Dr. Ruth K.M. Pfau Civil Hospital, Karachi Pakistan \\ 4 Contribution in the study \\ Instructor Radiology Department, The Aga Khan University Hospital, Karachi Pakistan \\ 5 Contribution in the study \\ 6 Assistant Professor, Department of Medicine Dow University of Health Sciences, Karachi Pakistan \\ Contribution in the study \\ CORRESPONDING AUTHOR \\ Dr. Reeta Rani \\ Assistant Professor, Department of Radiology, \\ Shaheed Mohtarma Benazir Bhutto Medical College \\ Lyari Pakistan \\ Email: chahatsachdev14@gmail.com \\ Submitted for Publication: 14-11-2020 \\ Accepted for Publication 19-03-2021

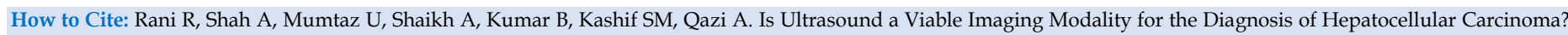 \\ A Cross-Sectional Study. APMC 2021;15(1):9-11. DOI: 10.29054/APMC/2021.1089
}

\section{ABSTRACT}

Background: Radiologists are always questioning the use of an imagine modality as compared to the other, this study helps answer that question for the diagnosis of Hepatocellular carcinoma. Objective: To compare the use of MRI imaging technique with ultrasonography for the diagnosis of hepatocellular carcinoma. Study Design: The type of study is a Prospective Cross-Sectional study. Settings: A large tertiary care hospital in Karachi, Pakistan. Duration: One year from July 2018 to July 2019. Methodology: The inclusion criteria was all the patients who were referred to us with symptoms for concern for hepatic tumors. For the MRI we used a 1.5 tesla machine, and for the ultrasound we used a conventional grey scale ultrasonography with a probe of $5 \mathrm{MHz}$. We performed multiphasic contrast enhanced MRI's, with images taken both before the injection of the contrast and afterwards in the various phases such as the arterial phase, portal venous phase and finally the delayed phase respectively. Results: The study population consisted of $n=110$ patients having a mean age of 46.5 $+/-5.50$ years. There were $n=78(70.90 \%)$ males and $n=32(20.09 \%)$ females. $N=101(91.81 \%)$ patients had a diagnosis of hepatocellular carcinoma as per MRI scan, for ultrasound $n=47(42.72 \%)$ patients were diagnosed as true positive, $n=2$ $(1.81 \%)$ were false positive, $n=54(49.09 \%)$ were false negative, while $n=7(6.36 \%)$ cases were truly negative. The specificity was $77.77 \%$ the sensitivity was $46.53 \%$, the positive predictive value was $95.91 \%$, negative predictive value was $11.4 \%$, and accuracy was $49.09 \%$ respectively. Conclusion: We found that the specificity of ultrasound as a diagnostic modality for hepatocellular carcinoma as compared to the MRI scan is good, however it is only able to correctly identify about half the patients, hence care should be taken when interpreting the results of ultrasound for hepatocellular carcinoma.

Keywords: Cirrhotic patients, Diagnostic accuracy, Magnetic resonance imaging, Hepatocellular carcinoma, Ultrasonography.

\section{INTRODUCTION}

Since the beginning of the $21^{\text {st }}$ century, there has been an increase in the prevalence of primary liver neoplasia's, and hepatocellular carcinoma being the most common of these primary liver tumors. Some authors consider it as the most common cause of cancer related mortality. ${ }^{1}$ Many authors believe that the morbidity of hepatocellular carcinoma can be decreased with an early detection and intervention. To that effect hepatobiliary magnetic resonance imaging (MRI) is considered a good choice as it provides good visuals for the regenerating and dysplastic nodules. Some authors believe that the computed tomography (CT) scan is a better option for diagnosis as compared to MRI.2,3 However proponents of the MRI imaging praise it by stating that it helps in the evaluation of cirrhosis and its complications in addition to the contrast injection which allows visualization of the blood vessels supplying the tumor. ${ }^{4}$ The image enhancement provided by the gadolinium contrast in a T1 weighted MRI scan helps in the detection and characterization of the lesions, especially when the hepatocellular carcinomas are smaller in size. ${ }^{2}$ The T2 weighted images help in the differentiation of the hepatocellular carcinoma from pre neoplastic lesions as well smaller early enhancing lesions. Radiologists believe that contrast enhanced MRI technique is much better than contrast enhanced CT scans, as the MRI technique allows the radiologists to use smaller amounts of contrast mediums and there is smaller incidences of acute kidney injuries and other contrast related reactions. Double 
hepatic arterial phase imaging has shown to provide less frequent off timed arterial phase imaging and a significant improvement in temporary resolution. ${ }^{3}$ There are various tissue specific contrast mediums which allow for a better visualization and characterization of liver pathologies.5,6 About $96 \%$ cases of hepatocellular carcinomas are accurately diagnosed with contrast enhanced MRI's while only $38 \%$ to $64 \%$ cases are diagnosed using ultrasound technique. $7,8,9$ However, Radiologists realize that low income and resource countries cannot afford expensive MRI imaging techniques, hence physicians in these countries often rely on cost effective imaging modalities. The aim of this current study is to compare the use of MRI imaging technique with ultrasonography for the diagnosis of hepatocellular carcinoma, as a conduit for better policy making for a resource poor country like Pakistan when it comes to the diagnosis of hepatocellular carcinomas.

\section{METHODOLOGY}

Study Design: Prospective cross-sectional study.

Settings: A large tertiary care hospital in Karachi, Pakistan.

Duration: One year from July 2018 to July 2019.

Sample Technique: Non-probability convenience sampling.

Sample Size: One hundred and ten patients.

Inclusion Criteria: The inclusion criteria was all the patients who were referred to the Department of Radiology, with symptoms of abdominal pain, weight loss, nausea and vomiting, fatigue, and accumulated fluid in the abdominal cavity, and any signs or symptom which the referring physicians considered a cause for concern for hepatic pathologies and tumors.

Exclusion Criteria: We excluded patients who were below the age of 18 years and refused to partake in the study.

Data Collection Procedure: All the patients signed an informed consent to participate in the study and the study was approved by the Departments Research Ethics Review Panel. The same MRI and ultrasound machines were used for all the patients. For the MRI we used a 1.5 tesla machine, and for the ultrasound we used a conventional grey scale ultrasonography with a probe of $5 \mathrm{MHz}$. We also performed multiphasic contrast enhanced MRI's, with images taken both before the injection of the contrast and afterwards in the various phases such as the arterial phase, portal venous phase and finally the delayed phase respectively. We took both $\mathrm{T} 1$ and T2 weighted images as well as fat suppression images. The images were taken in the coronal and transverse planes with slice thickness being $5 \mathrm{~mm}$, the slice gap being $1 \mathrm{~mm}$ and the flip angle being 75 degrees, the reconstruction matrix was set at 512 and the voxel size was set at $0.74 \mathrm{~mm}$ respectively. The element selections were 1234 and the pulse sequence parameters TR and TE were shortest in the T1 weighted images and the fat suppression images while it was the longest in T2 weighted images. All the scans and images were performed by the same resident radiologists for all the patients and were reassessed with a consultant radiologist for diagnostic accuracy. We used SPSS version 23.0 for windows for out statistical analysis and frequencies and percentages were used for our categorical variables and means and standard deviations were used for continuous variables. We collected all the data in a pre-designed proforma and made sure no identifying variables are used. We stored all the data in double secured space in our Department.

\section{RESULTS}

The study population consisted of $\mathrm{n}=110$ patients having a mean age of $46.5+/-5.50$ years, the age distribution is given in table 1 . There were $\mathrm{n}=78(70.90 \%)$ males and $\mathrm{n}=$ $32(20.09 \%)$ females. $\mathrm{N}=101(91.81 \%)$ patients had a diagnosis of hepatocellular carcinoma on the multiphasic MRI scan, the rest of the patients did not have hepatocellular carcinoma. Comparing the ultrasound findings with the MRI results, we observed that when we looked at the data from the ultrasonogram $n=47(42.72 \%)$ patients were diagnosed accurately (true positive) $n=2$ $(1.81 \%)$ patient was diagnosed as false positive, $n=54$ $(49.09 \%)$ patients were diagnosed as a case of hepatocellular carcinoma when they did not have the disease that is false negative while $n=7(6.36 \%)$ cases were truly negative. The specificity was $77.77 \%$ the sensitivity was $46.53 \%$, the positive predictive value was found to be $95.91 \%$, while negative predictive value was $11.4 \%$, and finally the diagnostic accuracy was found to be $49.09 \%$ respectively.

Table 1: Patients demographics and other variables for the study population of $n=110$ patients

\begin{tabular}{|c|l|c|c|}
\hline \multicolumn{2}{|c|}{ Variable } & Frequency & Percentage \\
\hline \multirow{4}{*}{ Age in years } & Between 15 to 30 years & 25 & $22.72 \%$ \\
\cline { 2 - 4 } & Between 31 to 60 years & 45 & $40.90 \%$ \\
\cline { 2 - 4 } & Between 61 to 80 years & 40 & $36.36 \%$ \\
\hline \multirow{2}{*}{ Gender } & Male & 78 & $70.90 \%$ \\
\cline { 2 - 4 } & Female & 32 & $20.09 \%$ \\
\hline
\end{tabular}

\section{DISCUSSION}

Our goal with this simple study was to see if ultrasound is a viable method for the diagnosis of hepatocellular carcinoma. The sensitivity of ultrasonography was found to be $46.53 \%$ and other similar studies have reported a sensitivity of 65 to $80 \%$ while they reported a specificity of greater than $90 \%$ while we found our specificity to be $77.77 \% .10,11,12$ The sensitivity for detecting smaller 
hepatocellular carcinomas using ultrasound is reported to be $42 \%$ for lesions that are less than $1 \mathrm{~cm}$ in size. ${ }^{13,14}$ For larger tumors the sensitivity is higher and close to $95 \% .{ }^{15}$ Radiologists have reported poor sensitivity of ultrasound especially in screening pre-transplant patients due to the anatomy and histology of the liver which provides it a coarse echotexture, similarly concomitant ascites also clouds the visibility thus reducing the sensitivity. ${ }^{16} \mathrm{~A}$ retrospective study by Bennett et al involving 200 patients who underwent screening prior to liver transplantation, the ultrasound data showed good correlation with explanted livers. They report a sensitivity between $13.5 \%$ to $50 \%$ depending on the diameter of the lesion. ${ }^{17}$ Radiologists recommend using either the MRI or the CT scan for liver transplant patients.

\section{CONCLUSION}

We found that the specificity of ultrasound as a diagnostic modality for hepatocellular carcinoma as compared to the MRI scan is good, however it is only able to correctly identify about half the patients, hence care should be taken when interpreting the results of ultrasound for hepatocellular carcinoma.

\section{LIMITATIONS}

There were several limitations to our study, first being the lack of differentiation according to the size of the tumor when it comes to measuring the sensitivity levels, secondly there is always a possibility of observation bias and sampling bias. Since we operate in a large publicly funded tertiary care hospital, it is quite common to see more severe cases at our institute. The sample size of the study was also quite small and not large enough to perform cost effectiveness analysis.

\section{SUGGESTIONS / RECOMMENDATIONS}

Our results do provide a backdrop for further studies to solidify the results. At the end we do agree that is resource poor countries, ultrasound may be the only diagnostic modality available to some hospitals and physicians and they should make the best use of the available resources while simultaneously opting to include MRI imaging machines to improve patients diagnosis, prognosis and satisfaction.

\section{CONFLICT OF INTEREST / DISCLOSURE}

None.

\section{ACKNOWLEDGEMENTS}

None.

\section{REFERENCES}

1. Ariff B, Lloyd CR, Khan S, Shariff M, Thillainayagam AV, Bansi DS et al. Imaging of liver cancer. World J Gastroenterol 2009;15(11):1289-1300.

2. Digumarthy SR, Sahani DV, Saini S. MRI in detection of hepatocellular carcinoma (HCC). Cancer Imaging. 2005;5(1):20-4.

3. Kanematsu M, Kondo H, Goshima S, Tsuge Y, Watanabe H. Magnetic Resonance Imaging of Hepatocellular Carcinoma. Oncology 2008;75(2):65-71.

4. Hussain SM, Reinhold C, Mitchell DG. Cirrhosis and lesion characterization at MR imaging. Radiographics 2009;29(6):1637-52.

5. Bellin MF. MR contrast agents, the old and the new. Eur J Radiol. 2006;60(3):314-23.

6. Gandhi SN, Brown MA, Wong JG, Aguirre DA, Sirlin CB. MR contrast agents for liver imaging: what, when, how. Radiographics. 2006;26(6):1621-36.

7. Hanna RF, Kased N, Kwan SW, Gamst AC, Santosa AC. Doublecontrast MRI for accurate staging of hepatocellular cancinoma in patients with cirrhosis. AJR Am J Roentgenol. 2008;190(1):47-57.

8. Singh P. EUS for detection of the hepatocellular carcinoma: results of a prospective study. Gastrointest Endosc 2007;66(2):265-73.

9. Tanaka S, Kitamura T. Recent advances in ultrasonographic diagnosis of hepatocellular carcinoma. Cancer. 1989;63(7):1313-7.

10. Bolondi L, Sofia S, Siringo S, Gaiani S, Casali A, Zironi G, Piscaglia F, Gramantieri L, Zanetti M, Sherman M. Surveillance programme of cirrhotic patients for early diagnosis and treatment of hepatocellular carcinoma: a cost effectiveness analysis. Gut 2001;48(2):251-9.

11. Bruix J, Sherman M. Management of hepatocellular carcinoma. Hepatology. 2005;42(2):1208-36.

12. Bruix J, Hessheimer AJ, Forner A, Boix L, Vilana R, Llovet JM. New aspects of diagnosis and therapy of hepatocellular carcinoma. Oncogene. 2006;25(27):3848- 56.

13. Dodd GD 3rd, Miller WJ, Baron RL, Skolnick ML, Campbell WL. Detection of malignant tumors in end-stage cirrhotic livers: efficacy of sonography as a screening technique. AJR Am J Roentgenol. 1992;159(4):727-33.

14. Franca AV, Elias Junior J, Lima BL, Martinelli AL, Carrilho FJ. Diagnosis, staging and treatment of hepatocellular carcinoma. Braz J Med Biol Res. 2004;37(11):1689-1705.

15. Colli A, Fraquelli M, Casazza G, Massironi S, Colucci A, Conte D, Duca P. Accuracy of ultrasonography, spiral CT, magnetic resonance, and alpha-fetoprotein in diagnosing hepatocellular carcinoma: a systematic review. Am J Gastroenterol. 2006;101(3):513-23.

16. Saar B, Kellner-Weldon F. Radiological diagnosis of hepatocellular carcinoma. Liver Int. 2008;28(3):189-99.

17. Bennett GL, Krinsky GA, Abitbol RJ, Kim SY, Theise ND, Teperman LW. Sonographic detection of hepatocellular carcinoma and dysplastic nodules in cirrhosis: correlation of pretransplantation sonography and liver explant pathology in 200 patients. AJR Am J Roentgenol. 2002;179(1):75-80.

18. Saab S, Ly D, Nieto J, Kanwal F, Lu D, Raman S, et al. Hepatocellular carcinoma screening in patients waiting for liver transplantation: a decision analytic model. Liver Transpl. 2003;9(7):672-81. 